\title{
THREE-DIMENSIONAL NUMERICAL SIMULATION OF OPERATION PROCESS IN ROTATING DETONATION ENGINE
}

\author{
S. M. Frolov, A. V. Dubrovskii, and V.S. Ivanov \\ Semenov Institute of Chemical Physics (ICP) \\ Moscow, Russia
}

The aim of this work was to create an efficient tool for transient threedimensional (3D) numerical simulation of the operation process in a Rotating Detonation Engine (RDE) with the particular emphasis to the design issues of the combustion chamber and isolators, thermal management and operation control. The governing equations are unsteady Reynolds-Averaged Navier-Stokes (URANS) equations coupled with a turbulence model and with the continuity and energy equations for a multicomponent reactive mixture. The algorithm used is the combination of Finite Volume Method and Particle Method recently developed at ICP to treat simultaneously frontal and volumetric combustion. The capabilities of the new numerical tool have been demonstrated for the annular cylindrical RDE operating on homogeneous stoichiometric hydrogenair mixture with a detonation rotation frequency of about 126,000 rpm. The calculations revealed considerable temperature and pressure pulsations at RDE inlet and outlet; however, special design adaptations were shown to allow their reduction.

\section{INTRODUCTION}

The concept of RDE put forward by Voitsekhovskii in 1959 [1] is very attractive for further developments in the field of air-breathing propulsion, in particular, for gas-turbine engines (GTE). The replacement of conventional combustors in a GTE by the annular rotating detonation chamber (RDC) and transition from the deflagration-to-detonation mode of combustion can theoretically lead to considerable gains in terms of propulsion unit efficiency and cost. As a matter of fact, in the detonation, the chemical reaction of fuel oxidation proceeds in the mode of self-ignition at high pressure behind a strong shock wave and is 3 to 4 orders of magnitude faster and, therefore, more energy efficient (estimated as $5 \%$

This is an Open Access article distributed under the terms of the Creative Commons Attribution License 2.0, which permits unrestricted use, distribution, and reproduction in any medium, provided the original work is properly cited. 
to $10 \%$ ) due to higher expansion potential of combustion products. In addition, due to shorter combustion time, the emissions of NOx and other pollutants in the detonation are expected to be lower.

The progress in the development of the RDC concept is mainly due to the long-term activity of the research team at Lavrentiev Institute for Hydrodynamics (see, e.g., $[2-5]$ ), whose accomplishments in experimental and numerical studies of rotating detonations are noteworthy. During the last decade, the interest to the concept has been growing worldwide: in France [6, 7], Poland [8], USA [9], Japan [10], and China [11].

The aim of this work was to create an efficient tool for transient 3D numerical simulation of the RDE operation process with the particular emphasis to design issues, thermal management, and operation control.

\section{ROTATION DETONATION ENGINE DESIGN CONSTRAINS}

It is implied that the GTE operating on rotating detonations has the architecture shown in Fig. 1. It contains an air intake, compressor, RDC, turbine, and nozzle. The main element - RDC - is the annular cylindrical combustor. The compressed air is continuously supplied to the RDC where it is mixed with fuel and the fuel-air mixture thus formed is burned in the rotating detonation wave. The detonation products pass the turbine and further expand in the nozzle. For damping the pressure waves emanating from the RDC, a provision should be made for gasdynamic isolators located both upstream and downstream from the RDC.

The RDC with the isolators is shown schematically in Fig. $2 a$. For the sake of simplicity, the RDC is assumed to operate on a homogeneous fuel-air mixture. The static pressure and temperature on the compressor side (on the bottom of upstream isolator) are kept constant and equal to $P_{\text {in }}$ and $T_{\text {in }}$, whereas the static pressure on the turbine side (at the RDC outlet) is controlled by the downstream isolator volume initially filled with air at a lower pressure $P_{\text {out }}<P_{\text {in }}$. Thus, the driving force for the mixture flow through the RDC in the absence of combustion is the pressure differential $\Delta P=P_{\text {in }}-P_{\text {out }}$. It is further assumed that from the compressor side, the fresh reactive mixture is supplied through the bottom

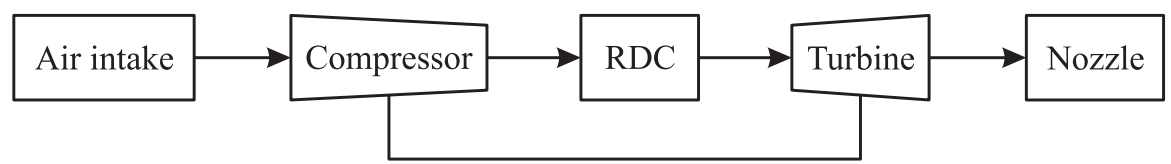

Figure 1 Architecture of the gas turbine engine operating on rotating detonations 


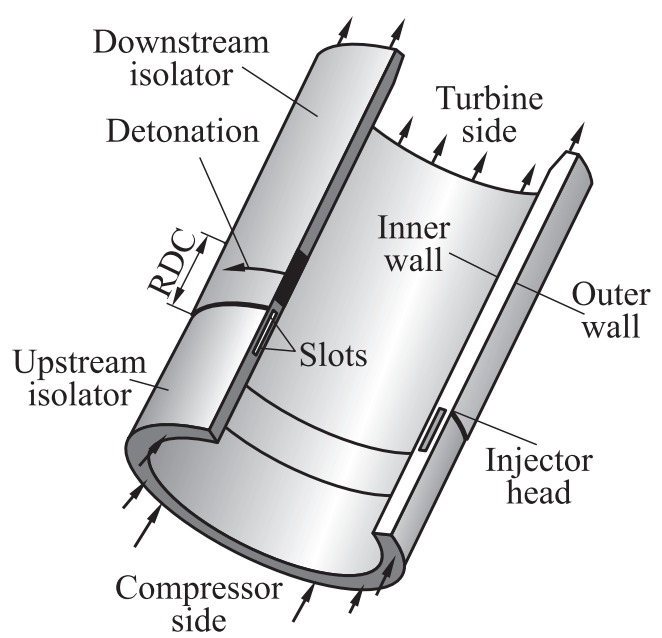

(a)

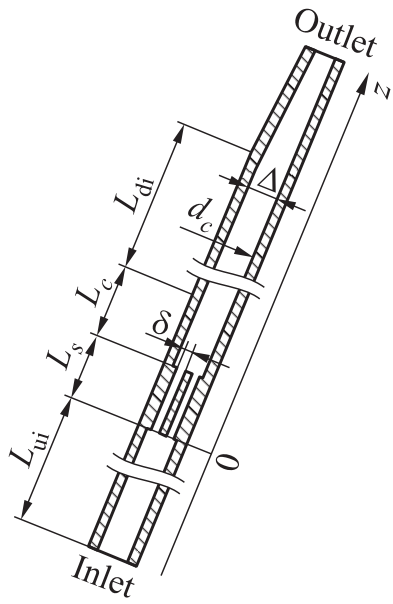

(b)

Figure 2 Rotating detonation chamber with upstream and downstream isolators $(a)$ and main geometrical dimensions with the longitudinal coordinate $z(b)$

annular gap to the upstream isolator of height $L_{\mathrm{ui}}$ and then to the annular combustion chamber of height $L_{c}$, outer wall diameter $d_{c}$, and gap width $\Delta$ through the injector face represented by two concentric slots of width $\delta$ and height $L_{s}$ (Fig. $2 b$ ). The combustion chamber is attached to the downstream isolator of height $L_{\mathrm{di}}$.

The detonation is directly initiated by a shock wave issuing from an initiator to the RDC filled with the reactive mixture. The self-sustained detonation wave, once initiated, propagates along the injector face, for example, in a counterclockwise direction (as shown in Fig. $2 a$ ), and burns the incoming mixture while the detonation products move downstream in the predominantly axial direction towards the turbine.

The detonation wave in the RDC exhibits several specific features.

First, it propagates in a semiconfined mixture layer: the layer is confined only by side walls (outer and inner walls of the RDC) whereas its bottom (injector head) is permeable and its top is unconfined. The conditions for the existence of such a detonation wave in terms of mixture composition, thermodynamic state, and geometrical dimensions of the layer are different from those for confined detonations in straight tubes. Moreover, the detonation parameters such as the propagation velocity, overpressure, etc. will be also different due to the strong effects of lateral expansion. 
Second, in the course of propagation, the detonation wave undergoes diffraction at the compressive outer wall and expansive inner wall of the RDC. This factor also affects the domain of detonation existence.

Third, its propagation direction is normal to the direction of high-speed turbulent jets of the incoming fresh mixture. As a result, the detonation wave tends to lift off and to lose the partial confinement from the bottom, thus getting weaker.

Fourth, the structure of the rotating detonation wave is expected to be somewhat different from the conventional cellular structure. The transverse waves inherent in the detonation front will interact with the compression and rarefaction waves generated by front diffraction at the outer and inner walls of the RDC, as well as with the waves generated by incoming jets of the fresh mixture.

Fifth, hot detonation products behind the propagating detonation wave partly mix with the newly injected fresh mixture causing either dilution of the latter or partial depletion due to relatively slow combustion or localized explosions giving birth to secondary shock waves and even detonations.

Sixth, the detonation is capable of penetrating through the slots in the injector face to the upstream isolator. To avoid it, the width of the slots in the injector face should be taken smaller than the minimum gap size for the detonation to go. However, the shock waves generated by the detonation are still capable of propagating upstream through the injector face to the isolator, thus disturbing the flow on the compressor side and affecting the injection of the fresh mixture into the RDC.

Seventh, due to continuous circulation of the detonation wave over the injector face, the inner and outer RDC walls can exhibit extremely high local thermal loads and cause premature ignition of the fresh mixture followed by detonation failure.

Note that in practical RDCs, the premixing of fuel and air in the upstream isolator will be prohibited for safety reasons. Therefore, an additional specific feature of the rotating detonation will come into play: the detonation will propagate over the injector head in the partly mixed reactants.

The phenomenology described above indicates the complexity of the flow field in the RDC. Clearly, the operation process with steadily rotating detonations is possible only when a set of requirements is met in terms of reactive mixture composition and thermodynamic parameters $P_{\text {in }}$ and $T_{\mathrm{in}}$, geometric dimensions of the chamber, upstream isolator, and injector head, as well as the material of the chamber wall and thermal conditions at the walls (cooling, etc.). In general, these requirements are not known a priori and the detonation existence domain should be found from problem solution. Nevertheless, the available knowledge on the dynamic parameters of detonations (detonation cell size, limiting diameter, limiting layer thickness, etc.) and detonability limits can be used as a starting point of the RDC design. 


\section{MATHEMATICAL MODEL}

The flow of a viscous compressible gas in the computational domain was described by the 3D URANS, energy conservation, and species continuity equations:

$$
\begin{aligned}
\rho \frac{d U_{i}}{d t} & =\rho \frac{\partial U_{i}}{\partial t}+\rho U_{j} \frac{\partial U_{i}}{\partial x_{j}}=-\frac{\partial P}{\partial x_{i}}+\frac{\partial}{\partial x_{j}}\left[\tau_{i j}-\rho \overline{U_{i}^{\prime} U_{j}^{\prime}}\right] ; \\
\rho \frac{d I}{d t} & =\rho \frac{\partial I}{\partial t}+\rho U_{j} \frac{\partial I}{\partial x_{j}}=\rho \dot{Q}+\frac{\partial P}{\partial t}+\frac{\partial}{\partial x_{j}}\left(\tau_{i j} U_{j}\right)+\frac{\partial}{\partial x_{j}}\left(\lambda \frac{\partial T}{\partial x_{i}}\right) ; \\
\rho \frac{d Y_{l}}{d t} & =\rho \frac{\partial Y_{l}}{\partial t}+\rho U_{j} \frac{\partial Y_{l}}{\partial x_{j}}=\rho \dot{r}_{l}+\frac{\partial}{\partial x_{j}}\left(\rho D_{l} \frac{\partial Y_{l}}{\partial x_{i}}-\rho \overline{Y_{l}^{\prime} U_{j}^{\prime}}\right)
\end{aligned}
$$

where $t$ is the time; $x_{j}(j=1,2,3)$ are the coordinates; $\rho$ is the mean density; $P$ is the mean pressure; $U_{i}$ is the mean velocity; $U_{i}^{\prime}$ is the fluctuating velocity component; $\tau_{i j}$ is the viscous stress tensor; $I=H+0.5 \sum_{i} U_{i}^{2}$ is the mean total enthalpy ( $H$ is the mean static enthalpy); $\lambda$ is the thermal conductivity; $T$ is the mean static temperature; $Y_{l}(l=1, \ldots, N)$ is the mean mass fraction of the $l$ th species of the mixture ( $N$ is the total number of species in the mixture); $D_{l}$ is the molecular diffusion coefficient of species $l ; Y_{l}^{\prime}$ is the fluctuation of the mass fraction of the $l$ th species, and $\dot{r}_{l}$ and $\dot{Q}$ are the mean source terms of species mass and energy (e.g., chemical reactions).

The turbulent fluxes of mass, momentum, and energy in Eqs. (1)-(3) were modeled using the standard $k-\varepsilon$ turbulence model. Here, $k$ is the kinetic energy of turbulence, and $\varepsilon$ is its dissipation.

In general, modeling of the chemical sources $\dot{r}_{l}$ and $\dot{Q}$ for turbulent combustion and detonation requires taking into account the contributions from both frontal combustion (index $f$ ) and volumetric reactions (index $V$ ):

$$
\dot{r}_{l}=\dot{r}_{i f}+\dot{r}_{l V} ; \quad \dot{Q}=\dot{Q}_{f}+\dot{Q}_{V} .
$$

However, in view of the specific features of the phenomena described above, here, the contribution of frontal combustion to the chemical sources is neglected assuming $\dot{r}_{l f}=0$ and $\dot{Q}_{f}=0$. The contributions from volumetric reactions $\dot{r}_{l V}$ and $\dot{Q}_{V}$ were determined using the Particle Method (PM) [12-15].

In the PM algorithm, the instantaneous local states of a turbulent reacting flow are presented as a set of interacting notional (Lagrangian) particles. Each $i$ th particle has individual properties: the position in space $x_{k}^{i}$ and three local instantaneous velocity components $u_{k}^{i}(k=1,2,3)$, volume $V^{i}$, density $\rho^{i}$, the static enthalpy $h^{i}$, mass fractions of species $y_{l}^{u}(l=1, \ldots, N)$, and the statistical weight $w^{i}$ used in determining the mean values of the variables over the ensemble of particles. For each $i$ th particle, the following system of equations is solved [12]: 


$$
\begin{aligned}
\frac{d x_{k}^{i}}{d t} & =u_{k}^{i} ; \\
\frac{d\left(\rho_{l}^{i} V^{i}\right)}{d t} & =\nabla J_{l}^{i}+J_{l h}^{i} ; \\
\rho^{i} \frac{d u_{k}^{i}}{d t} & =\frac{\partial P^{i}}{\partial x_{k}}-\nabla \tau^{i} ; \\
\rho^{i} \frac{d h^{i}}{d t} & =-\nabla q^{i}+\rho^{i} \dot{Q}_{V}^{i}+\frac{\partial P^{i}}{\partial t}-P^{i} \frac{\partial u_{k}^{i}}{\partial x_{k}}
\end{aligned}
$$

where $\rho_{l}^{i}=y_{l}^{i} \rho^{i}$ is the partial density of the $l$ th species in the $i$ th particle; $J_{l h}^{i}$ is the change of the mass concentration of the $i$ th species in the course of chemical reactions; $P^{i}$ is the mean static pressure at the point of location of the $i$ th particle; $\dot{Q}_{V}^{i}$ is the rate of energy release by chemical reactions in the $i$ th particle; $\nabla J_{l}^{i}$ is the diffusion flux of the $l$ th species to the $i$ th particle; $\nabla \tau^{i}$ is the momentum flux to the $i$ th particle due to molecular viscosity; and $\nabla q^{i}$ is the heat flux to the $i$ th particle.

To determine the flux (exchange) terms in the PM, the classical model of linear decay to the mean is used [12]:

$$
\begin{aligned}
\nabla J_{l}^{i} & =-0.5 C\left(y_{l}^{i}-Y_{l}^{i}\right) \rho^{i} V^{i} \omega ; \\
\left(\rho^{i}\right)^{-1} \nabla\left(p^{i} E-\tau^{i}\right) & =-\xi\left(u_{k}^{i}-U_{k}^{i}\right)+A(t) ; \\
\nabla q^{i} & =-0.5 C\left(h^{i}-H^{i}\right) \rho^{i} \omega
\end{aligned}
$$

where $C \approx 2.0$ and $\zeta \approx 2.075 \omega$ are the coefficients; $Y_{l}^{i}, U_{k}^{i}$, and $H^{i}$ are the mean mass fraction of $l$ th species, mean velocity, and mean enthalpy of the gas at the point of location of the $i$ th particle, respectively; $p^{i}$ is the pulsating pressure component, $\omega=\varepsilon / k$ is the frequency of turbulent fluctuations; and $A(t)=\left(C_{0} \varepsilon\right)^{1 / 2} d W(t) / d t$ is the random function describing the influence of fluctuations of pressure and velocity on the motion of the particle; here, $C_{0} \approx 2.1$ is a continuous random variable having normal distribution and satisfying the condition $\overline{d W_{i}(t)}=0$ and $\overline{d W_{i}(t) d W_{j}(t)}=\delta_{i j} d t$ ( $\delta_{i j}$ is the Kronecker symbol). The mean quantities $Y_{l}^{i}, U_{k}^{i}$, and $H^{i}$ are determined either by interpolating the corresponding values of $Y_{l}, U_{k}$, and $H$ (obtained from the solution of averaged Eqs. (1)-(3)) or by ensemble averaging over particles in a computational cell using the formulas:

$$
Y_{l}^{i}=\sum_{i} w^{i} y_{l}^{i} ; \quad U_{k}^{i}=\sum_{i} w^{i} u_{k}^{i} ; H^{i}=\sum_{i} w^{i} h^{i}
$$

where the statistical weight of the $i$ th particle is given by

$$
w^{i}=\frac{\rho^{i} V^{i}}{\sum_{i} \rho^{i} V^{i}} .
$$


System (1)-(3) in conjunction with the $k-\varepsilon$ turbulence model and the PM were closed by the caloric and thermal equations of state of an ideal gas with variable specific heat and supplemented by the initial and boundary conditions. All the thermophysical parameters of the gas were considered variable.

The mean pressure field $P\left(t, x_{k}\right)$ and the local frequency of turbulent fluctuations required for solving the system of Eqs. (4)-(7) supplemented by additional relations (8)-(10), are determined by solving the averaged Eqs. (1)-(3) and the equations of the $k-\varepsilon$ turbulence model.

The most important advantage of the PM is the possibility of accurately determining the rates of chemical reactions in a turbulent flow: the source terms $J_{l h}^{i}$ and $\dot{Q}_{V}^{i}$ are determined using the known mechanisms of chemical reactions as well as the instantaneous mass fractions $y_{l}^{i}(l=1, \ldots, N)$ and temperature $\theta^{i}$ in the $i$ th particle. The instantaneous local rate of change of the mass concentration of the $l$ th species in the $i$ th particle is calculated by the formula:

$$
J_{l h}^{i}=V^{i} W_{l} \sum_{k=1}^{L}\left(\nu_{l, k}^{\prime \prime}-\nu_{l, k}^{\prime}\right) A_{k}\left(\theta^{i}\right)^{n_{k}} \exp \left(-\frac{E_{k}}{R \theta^{i}}\right) \prod_{j=1}^{N}\left(\frac{y_{j}^{i} \rho^{i}}{W_{j}}\right)^{\nu_{j, k}^{\prime}}
$$

where $W_{l}$ is the molecular mass of the $l$ th species; $\nu_{l, k}^{\prime}$ and $\nu_{l, k}^{\prime \prime}$ are the stoichiometric coefficients for the $l$ th species acting as a reagent or product in the $k$ th reaction, respectively; $A_{k}, n_{k}$, and $E_{k}$ are the preexponential factor, the temperature exponent, and the activation energy for the $k$ th reaction; $R$ is the universal gas constant; $L$ and $N$ are, respectively, the total numbers of reactions and species in the chemical mechanism.

The rate of energy release by chemical reactions in the $i$ th particle is calculated by the formula:

$$
\dot{Q}_{V}^{i}=\frac{1}{\rho} \sum_{k=1}^{L} H_{k} A_{k}\left(\theta^{i}\right)^{n_{k}} \exp \left(-\frac{E_{k}}{R \theta^{i}}\right) \prod_{j=1}^{N}\left(\frac{y_{j}^{i} \rho^{i}}{W_{j}}\right)^{v_{j, k}^{\prime}}
$$

where $H_{k}$ is the heat effect of the $k$ th chemical reaction.

Knowing $J_{l h}^{i}$ and $\dot{Q}_{V}^{i}$, one can determine the contribution of bulk reactions $\dot{r}_{l V}$ and $\dot{Q}_{V}$ to the chemical source terms $\dot{r}_{l}$ and $\dot{Q}$

$$
\dot{r}_{l V}=\frac{\sum_{i} w^{i} J_{l h}^{i}}{\sum_{i} \rho^{i} V^{i}}, \quad \dot{Q}_{V}=\sum_{i} w^{i} \dot{Q}_{V}^{i}
$$




\section{COMPUTATIONAL APPROACH AND MAIN PARAMETERS}

The algorithm used for solving the governing equations was the combination of semiimplicit method for pressure-linked equations (SIMPLE) with the semiimplicit Monte Carlo method for notional particles. The chemical source terms were obtained using implicit numerical algorithm with internal time stepping. The combined algorithm has been thoroughly validated against experimental data on flame acceleration and deflagration-to-detonation transition in tubes with and without obstacles [14], as well as for shock-induced ignition and preflame autoignition in enclosures [16].

The fuel used was hydrogen. Only the stoichiometric hydrogen-air composition was considered in this particular study. For modeling hydrogen oxidation, a simplified single-step reaction mechanism was applied:

$$
\mathrm{H}_{2}+\mathrm{H}_{2}+\mathrm{O}_{2} \rightarrow \mathrm{H}_{2} \mathrm{O}+\mathrm{H}_{2} \mathrm{O} .
$$

As reaction (11) does not account for water dissociation, its heat effect was properly modified to fit the theoretical Chapman-Jouguet (CJ) detonation velocity $(\sim 1970 \mathrm{~m} / \mathrm{s})$. The rate of hydrogen oxidation $\left[\dot{\mathrm{H}}_{2}\right]$ at elevated initial pressures $P$ (from 5 to $40 \mathrm{~atm}$ ) and temperatures $T$ (from 1100 to $2000 \mathrm{~K}$ ) was calculated according to the simple relationship:

$$
\left.\left[\dot{\mathrm{H}}_{2}\right]=-8.0 \cdot 10^{11} P^{-1.15}\left[\mathrm{H}_{2}\right]^{2}\left[\mathrm{O}_{2}\right] e^{-10,000 / T} \text { (atm, mole, } \mathrm{l}, \mathrm{s}\right) .
$$

This relationship was obtained by fitting the ignition delays predicted by the validated detailed reaction mechanism [17] with that provided by reaction (11).

The main governing parameters used in the calculations and five test cases considered herein are listed in Table 1 . The height of the RDC, $L_{c}$, is taken equal to $100 \mathrm{~mm}$ to allow for the formation of the detonable mixture layer approximately equal to the critical height [3] $h_{m}=(12 \pm 5) \lambda_{D}$ where $\lambda_{D}$ is the detonation cell width at a certain mean pressure in the RDC. As this pressure is a priori unknown, for the sake of estimation, the value of $\lambda_{D}$ was taken for normal initial conditions $\left(\lambda_{D} \approx 15 \mathrm{~mm}\right.$ for the stoichiometric hydrogen-air mixture at $0.1 \mathrm{MPa}$ and $298 \mathrm{~K})$. Geometrical parameters $d_{c}$ and $\Delta$ entering Table 1 were chosen according to [5]. Following [3], the parameters $h_{m}, d_{c}$, and $\Delta$ determine the total number $n_{D}$ of detonation waves capable of propagating in the RDC: $n_{D}=\pi d_{a} /\left((7 \pm 2) h_{m}\right)$ where $d_{a}=d_{c}-\Delta$ is the average diameter of the RDC. Clearly, for the adopted values of $L_{c} \approx h_{m}, d_{c}$, and $\Delta$, only one detonation wave can propagate in the RDC, which could simplify the analysis. Note again that all these estimates are used as a starting point of the RDC design and the actual operation mode of the combustor can be obtained only in the course of the problem solution. 
Table 1 Main parameters of calculations and test cases

\begin{tabular}{ccccccc}
\hline$L_{\mathrm{ui}}, \mathrm{mm}$ & $L_{s}, \mathrm{~mm}$ & $L_{c}, \mathrm{~mm}$ & $L_{\mathrm{di}}, \mathrm{mm}$ & $d_{c}, \mathrm{~mm}$ & $\Delta, \mathrm{mm}$ & $\delta, \mathrm{mm}$ \\
\hline 200 & 50 & 100 & 450 & 306 & 23 & 6.9 \\
\hline \hline Test case & $P_{\text {in }}$, atm & $T_{\text {in }}, \mathrm{K}$ & $T_{w}, \mathrm{~K}$ & $k_{\text {in }}, \mathrm{J} / \mathrm{kg}$ & $\varepsilon_{\text {in }}, \mathrm{J} /(\mathrm{kg} \cdot \mathrm{s})$ & $Y_{\mathrm{H}_{2}, \text { in }}$ \\
\hline 1 & 15 & 293 & 293 & $10^{-3}$ & $2 \cdot 10^{-4}$ & 0.028 \\
2 & 20 & 293 & 293 & $10^{-3}$ & $2 \cdot 10^{-4}$ & 0.028 \\
3 & 10 & 293 & 293 & $10^{-3}$ & $2 \cdot 10^{-4}$ & 0.028 \\
4 & 25 & 293 & 293 & $10^{-3}$ & $2 \cdot 10^{-4}$ & 0.028 \\
5 & 10 & 580 & 293 & $10^{-3}$ & $2 \cdot 10^{-4}$ & 0.028 \\
$5^{*}$ & 10 & 580 & 293 & $10^{-3}$ & $2 \cdot 10^{-4}$ & 0.028 \\
\hline
\end{tabular}

The boundary conditions for the mean flow velocity, pressure, total enthalpy, turbulent kinetic energy and its dissipation, and species concentrations at the rigid walls of the computational domain were formulated assuming impermeable, no-slip, noncatalytic, isothermal walls at temperature $T_{w}$ and using the approach of wall functions.

At the compressor side, the inlet conditions (index 'in') in terms of fixed $P_{\text {in }}$, $T_{\text {in }}, k_{\text {in }}, \varepsilon_{\text {in }}$, and $Y_{l, \text { in }}(l=1, \ldots, N)$ were specified. At the turbine side, the outlet conditions (index 'out') in terms of either fixed $P_{\text {out }}$ or $\partial P_{\text {out }} / \partial z=0$ were set. In both cases, a relatively large downstream isolator volume was attached to the $\mathrm{RDC}$ to avoid the effect of reflected disturbances on the RDC operation process. As a matter of fact, the computational domain was shaped in such a way that the outlet boundary conditions did not affect the solution, i. e., the flow velocity in the downstream isolator was supersonic. All other variables (velocity, total enthalpy, turbulent kinetic energy and its dissipation, and species concentrations) were extrapolated from internal computational cells to the outlet cross section.

The boundary conditions for notional particles (velocity and scalar properties) at rigid walls, inlet, and outlet were formulated in a way consistent with the boundary conditions for the mean values of the corresponding variables. This consistency was continuously controlled by comparing the values of the variables obtained by ensemble averaging over all particles in a computational cell with their mean values.

Initially, the upstream isolator and RDC were assumed to be filled with the quiescent reactive mixture, whereas the downstream isolator was filled with pure air. To initiate a detonation, a provision was made for a finite-size domain containing the combustion products of the stoichiometric hydrogen-air mixture $\left(\mathrm{H}_{2} \mathrm{O}\right.$ and $\left.\mathrm{N}_{2}\right)$ at elevated pressure and temperature.

The spatial positions of particles $x_{k}^{i}(k=1,2,3)$ were initialized stochastically all throughout the computational domain. Each particle was initially provided with the values of $u_{k}^{i}, V^{i}, \rho^{i}, h^{i}, y_{l}^{i}(l=1, \ldots, N)$, and $w^{i}$ consistent with the 
initial distributions of mean variables. The nominal number of particles in each cell, $N_{p}$, was a preset value. In the calculations presented below, $N_{p}=10$. Note that in the course of calculations, the actual number of particles in cells can change. To keep the number of particles at a preset level, the proper procedures of particle clustering and cloning were used.

For the sake of simplicity, in this particular study, the particles evolved in the flow field according to Eqs. (4) to (7) with the mean quantities $Y_{l}^{i}, U_{k}^{i}$, and $H^{i}$ determined by interpolating the $Y_{l}, U_{k}$, and $H$ values obtained from the solution of averaged Eqs. (1)-(3). Furthermore, to speed up the calculations, a special ignition triggering procedure was introduced: when the rate of temperature raise $\dot{\theta}^{i}$ in at least one particle in cell exceeded a prescribed threshold value $\dot{\theta}_{\text {ign }}^{i}$ $=10^{7} \mathrm{~K} / \mathrm{s}$, the reactive mixture in this cell was forced to be burned within a single time step. For the conditions behind a detonation wave, this approach proved to be well justified because the sensitivity of the computational results to the required number of particles in cell simultaneously reaching this ignition threshold was very low.

In order to force the detonation wave propagating in a specified direction (e.g., counterclockwise), initially, a provision was made for a layer of temporarily nonreacting particles (with $J_{l h}^{i}=0$ ) in the clockwise direction from the initiation domain. Shortly after initiation, these particles became reacting. The flow pattern in the RDC can be expected to depend on the number of notional particles. However, when the mean number of particles per computational cell was sufficiently large $\left(N_{p}>10-15\right)$, this dependence was becoming weak.

The effect of computational grid on the flow pattern in the RDC is also noteworthy. The results presented herein were obtained at the computational grids insufficient for the detailed resolution of the detonation structure but proved to be good enough to determine the detonation existence domain and to capture the main physical and chemical phenomena inherent in the operation process.

\section{RESULTS OF CALCULATIONS}

Figure 3 shows the snapshots of static temperature $(a)$, total pressure $(b)$, hydrogen mass fraction $(c)$ and heat flux to the outer wall $(d)$ at two time instants separated by time interval $\Delta t=60 \mu$ s for test case 1 with $P_{\text {in }}=1.5 \mathrm{MPa}$. All the distributions are taken in the close vicinity to the rigid walls of the computational domain. In this test case, the operation process in the RDC with a single detonation wave has attained the limiting cycle conditions after 3-4 rotations of the wave, i. e., upon 1-2 ms after detonation initiation. The rotation frequency of the detonation wave was $126,000 \mathrm{rpm}(2.1 \mathrm{kHz})$. One can see that the height of the layer immediately ahead of the curved detonation front (marked in red in Figs. $3 a$ and $3 b$ ) is about $80 \mathrm{~mm}$, which is close to the critical height $h_{m}$ esti- 

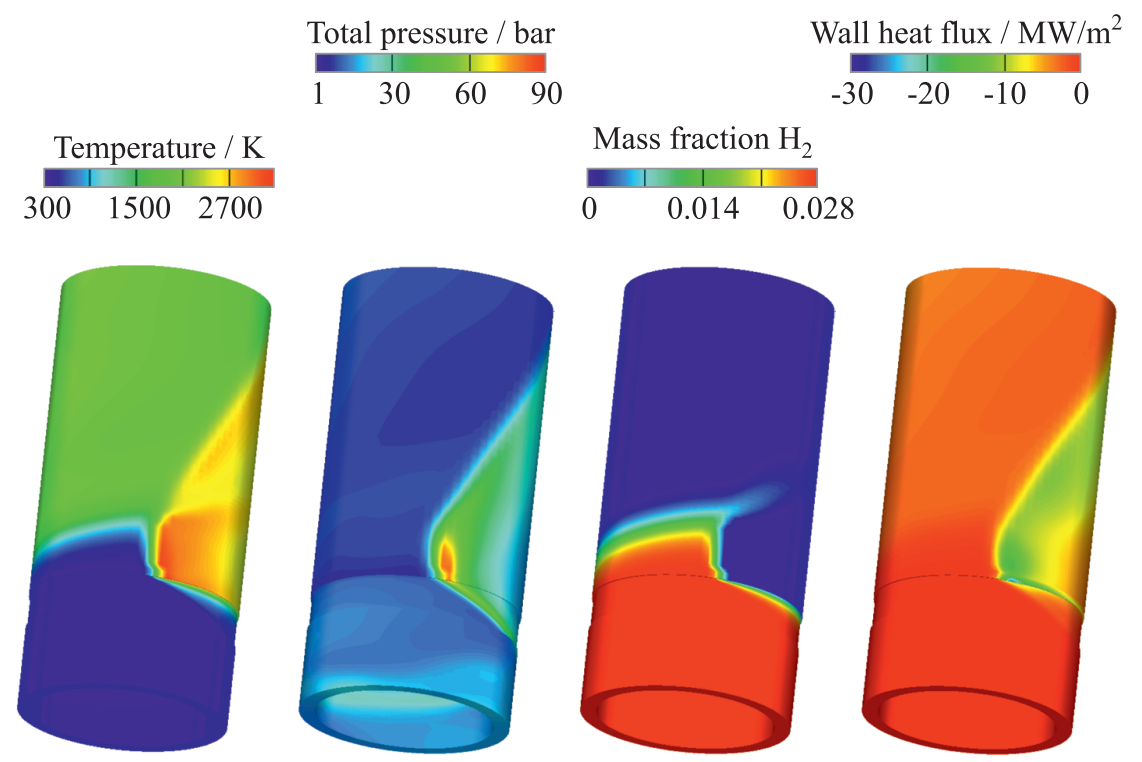

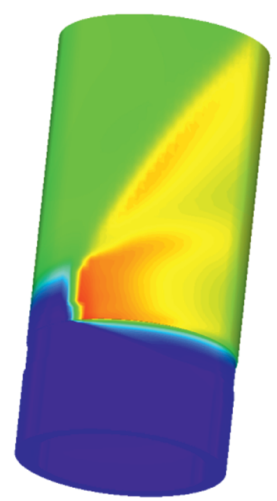

(a)

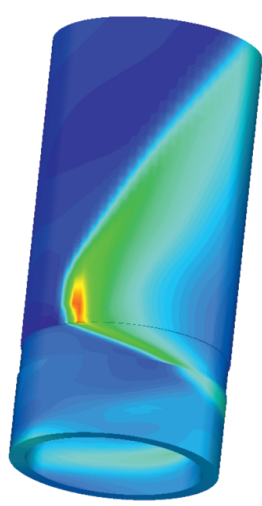

(b)

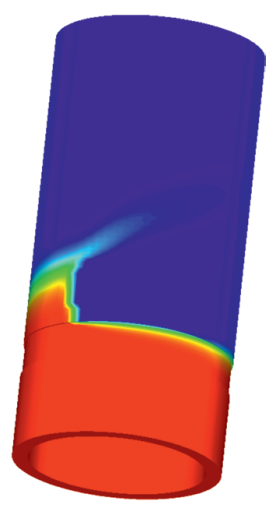

(c)

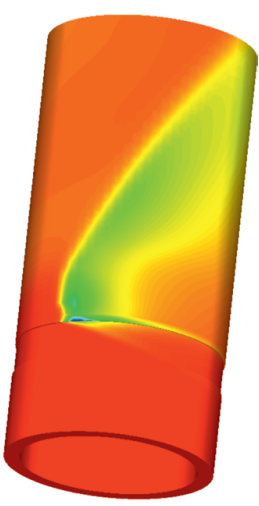

(d)

Figure 3 Snapshots of mean static temperature $(a)$, total pressure $(b)$, hydrogen mass fraction $(c)$, and heat flux to the wall $(d)$ at $t=5.75$ (top) and $5.81 \mathrm{~ms}$ (bottom) for test case 1 .

mated by the empirical criterion [3] despite the mean static pressure in the RDC is higher than $0.1 \mathrm{MPa}$ (see above). The attempts to initiate simultaneously two or more detonation waves in the RDC of the chosen size failed. In all cases, only the mode with a single detonation wave was stable in a long run. This fact is roughly in line with the empirical criterion [3] for the total number of detonation 
waves capable of propagating simultaneously in the RDC of the chosen geometry $\left(n_{D}=\pi d_{a} /\left((7 \pm 2) / h_{m}\right) \approx 3.14 \cdot 283 /(7 \pm 2) / 80=1.2-2.2\right)$ (see section 4 above). The shock waves generated by the detonation propagate both upstream and downstream (see Fig. 3b). Fuel is burned in the RDC completely: a tail of fuel not reacted in the detonation wave (see Fig. $3 c$ ) disappears at a half-height of the downstream isolator. The local heat flux to the outer wall (Fig. $3 d$ ) attains extremely high values on the level of $16 \mathrm{MW} / \mathrm{m}^{2}$ near the injector head. It is interesting that the hot detonation products penetrate only partly to the slots of the injector head and do not penetrate at all into the upstream isolator. Note that the contribution of dynamic pressure to the total pressure at the upstream isolator inlet (compressor side) is relatively small (less than $3 \% P_{\text {in }}$ ).

The operation parameters of the RDC in the course of 12 successive rotations of the detonation wave are presented in Fig. 4. Shown in Fig. 4 are the time histories of static pressure $P$ (Fig. $4 a$ ) and static temperature $T$ (Fig. $4 b$ ) in the point at $z=20 \mathrm{~mm}$ above the injector head in the middle of RDC gap $\Delta$. For the sake of comparison, two sets of time histories are plotted in Fig. 4: one for test case 1 (marked in black) and the other for test case 2 (marked in grey). Dashed lines in Fig. $4 a$ show the static pressure averaged over the RDC upon achieving the limit cycle conditions in the both test cases. The static pressure averaged over the RDC is seen to depend on $P_{\text {in }}$ : the higher $P_{\text {in }}$, the higher the pressure. The static pressures averaged over the RDC are 1.44 and $1.94 \mathrm{MPa}$, respectively, and are somewhat lower than the corresponding values of $P_{\text {in }}$. Peak-to-peak variations in the static pressure and temperature for successive detonation waves are obviously caused by the multidimensional nature of the detonation and its propagation in a cylindrical gap accompanied with diffraction phenomena. The difference between the variations of peak static

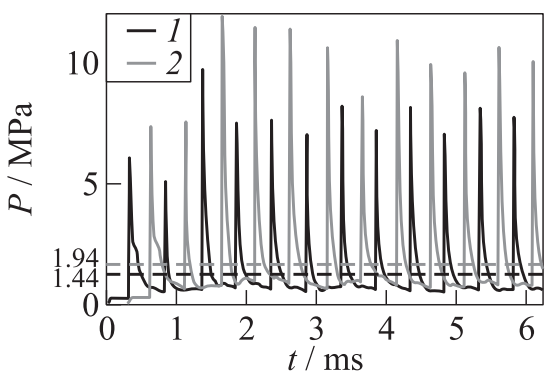

(a)

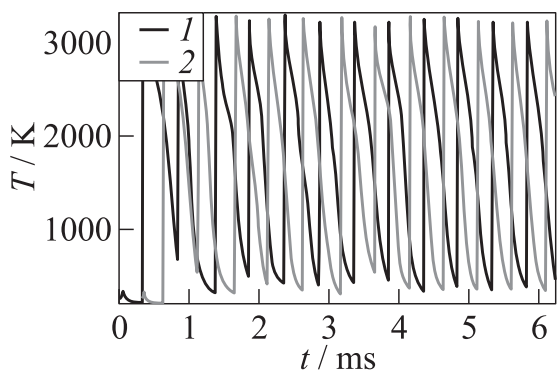

(b)

Figure 4 Time histories of static pressure $(a)$ and static temperature $(b)$ at the point located $20 \mathrm{~mm}$ above the injector head in the middle of the RDC gap for test cases 1 (1) and 2 (2). Dashed curves show the static pressure averaged over the RDC upon achieving the limit cycle conditions 


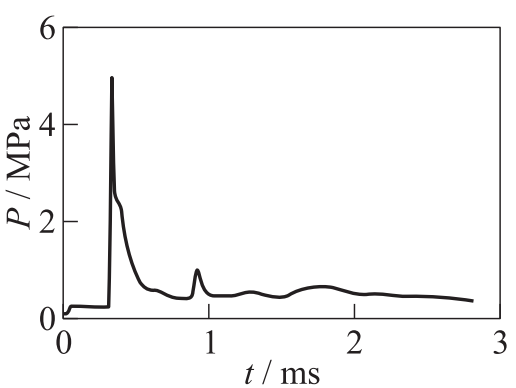

(a)

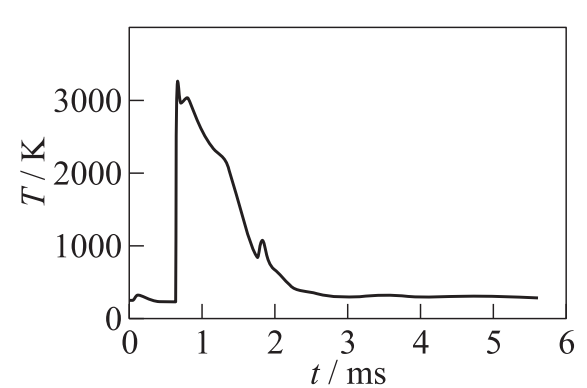

(b)

Figure 5 Time histories of static pressure $(a)$ and static temperature $(b)$ at the point located $20 \mathrm{~mm}$ above the injector head in the middle of the RDC gap for test case 3 with detonation failure

pressure and peak temperature in the successive waves is most probably caused by the adopted simplified ignition triggering procedure based on the threshold value $\dot{\theta}_{\text {ign }}^{i}$ rather than on continuous solution of kinetic equations for all particles in cell up to reaction completion.

It is important that the values of total pressure averaged over the RDC are higher than $P_{\text {in }}$, namely, 1.71 and $2.35 \mathrm{MPa}$ for test cases 1 and 2, respectively. This means that there are approximately $11 \% P_{\text {in }}$ and $14 \% P_{\text {in }}$ gains in total pressure in these test cases. That is why the RDC is often referred to as the Pressure Gain Combustor. Contrary to the static pressure, the static temperature seems to be independent of the injection pressure (see Fig. $4 b$ ). The mean detonation velocity in both test cases is approximately $1850 \mathrm{~m} / \mathrm{s}$ which is $6 \%$ lower than the CJ detonation velocity for the stoichiometric hydrogen-air mixture at normal initial conditions $(\sim 1970 \mathrm{~m} / \mathrm{s})$.

The authors' attempts to obtain a stable operation process at $P_{\text {in }}<1.3 \mathrm{MPa}$ and $P_{\text {in }}>2.5 \mathrm{MPa}$, other conditions being similar to those in test cases 1 and 2, failed. For example, at $P_{\text {in }}=1.0 \mathrm{MPa}$, the detonation once initiated was decaying as shown in Fig. 5. At $P_{\text {in }}>2.5 \mathrm{MPa}$, the detonation failure was caused by too high mass flow rates through the injector head. Moreover, severe premature ignitions in the tail of the detonation wave were observed in the calculations due to mixing of incoming fresh reactants with hot combustion products. Thus, the domain of detonation existence for this particular set of governing parameters was $1.3 \leq P_{\text {in }} \leq 2.5 \mathrm{MPa}$.

Figure 6 demonstrates that different boundary conditions at the turbine side (Fig. $6 a$ ) do not affect the results of calculations. As a matter of fact, the time histories of local static pressure (and other variables) in the RDC are identical for the conditions of fixed pressure $P_{\text {out }}=0.1 \mathrm{MPa}$ and zero pressure gradient $\partial P_{\text {out }} / \partial z=0$ (Fig. $\left.6 b\right)$. 


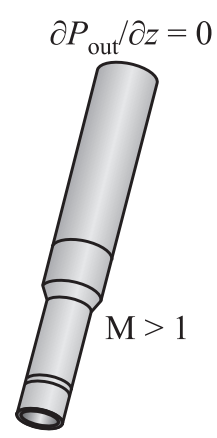

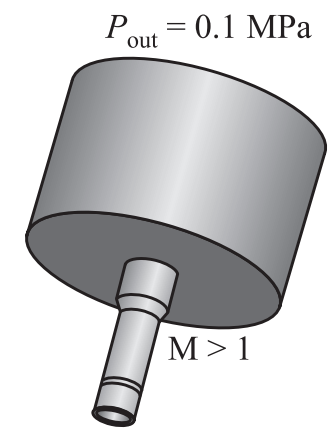

(a)

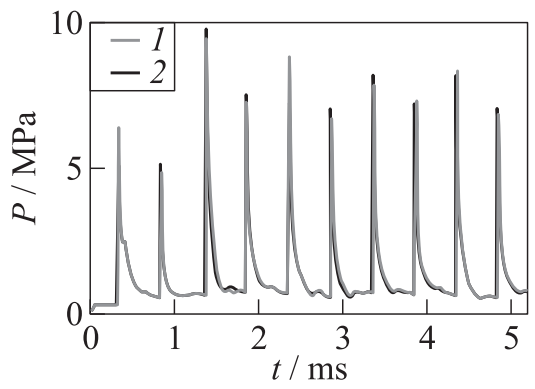

(b)

Figure 6 Different arrangements for outlet boundary conditions $(a)$; and time histories of static pressure in the point located $20 \mathrm{~mm}$ above the injector head obtained with boundary conditions $P_{\text {out }}=0.1 \mathrm{MPa}(1)$ and $\partial P_{\text {out }} / \partial z=0(2)(b)$

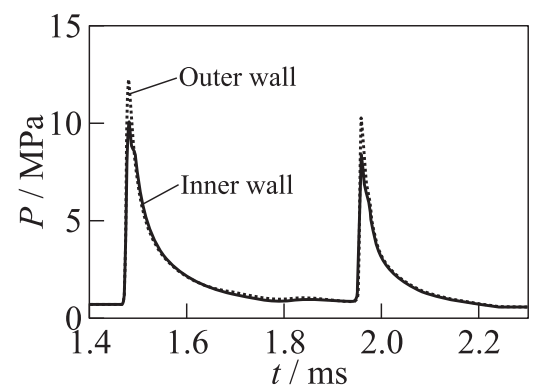

(a)

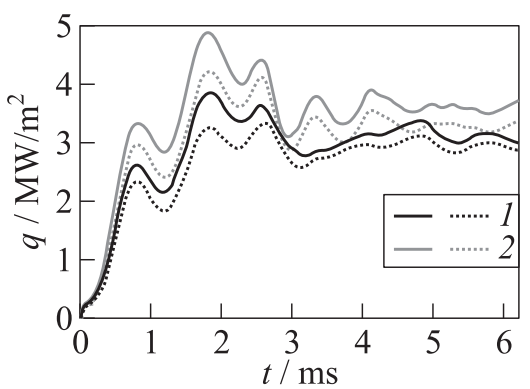

(b)

Figure 7 Time histories of the static pressure at the inner (solid curve) and outer (dotted curve) walls of the RDC in the vicinity to the injector head in test case $1(a)$ and of the total heat fluxes to the inner (solid curves) and outer (dotted curves) walls of the RDC with attached downstream isolator in test cases $1(1)$ and $2(2)(b)$

Figure $7 a$ further demonstrates the specific features of the detonation wave in the vicinity to the injector head in terms of the time histories of the local static pressure at the outer (dotted curve) and inner (solid curve) walls of the RDC. It follows from Fig. $7 a$ that due to detonation wave diffraction, the peak static pressure at the inner and outer walls of the RDC differs by about $20 \%-25 \%$, whereas the subsequent pressure evolution is nearly identical at both walls.

Figure $7 b$ shows the time histories of the total heat flux to the inner (solid curves) and outer (dotted curves) walls of the RDC with the attached down- 


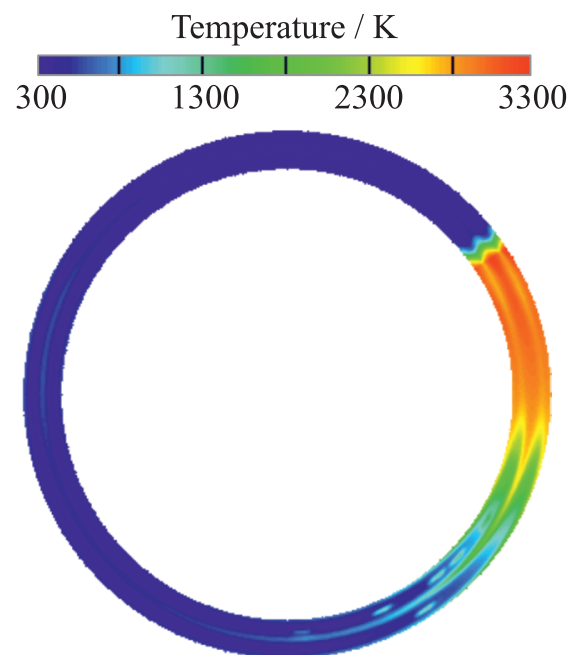

(a)

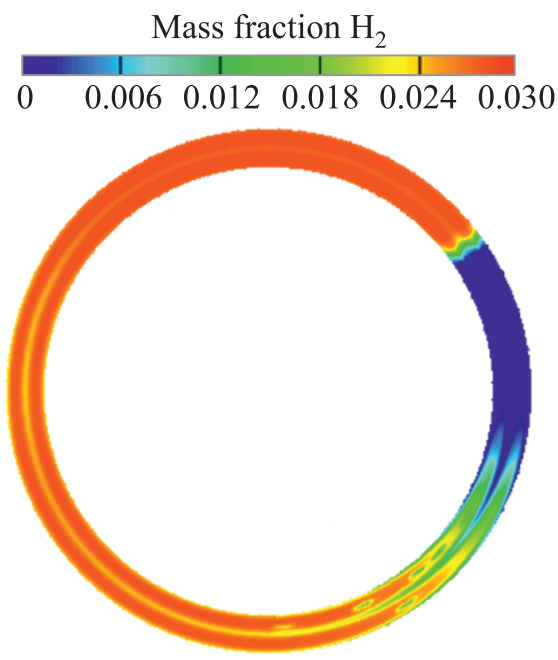

(b)

Figure 8 Snapshots of static temperature $(a)$ and hydrogen mass fraction $(b)$ in the $\mathrm{RDC}$ cross section at height $z=5 \mathrm{~mm}$ above the injector head in test case 4 .

stream isolator for test cases 1 (curves 1 ) and 2 (curves 2). The total heat fluxes were defined as the integral-mean values over inner or outer wall surfaces. One can see that after several initial oscillations, the total heat fluxes tend to level out. This is caused by the fact that the RDC is gradually (within several cycles) getting filled with hot detonation products (displacing initial air) and the contribution of extremely high local heat fluxes in the thin detonation front to the integral-mean values gradually decreases. The inner wall is seen to exhibit higher total heat fluxes than the outer wall. The difference between these heat fluxes is on the level of $5 \%$ for test case 1 and $10 \%$ for test case 2 . The total heat flux to the RDC walls increases with $P_{\text {in }}$. Thus, the mean value of the total heat flux (to both RDC walls) is about $3 \mathrm{MW} / \mathrm{m}^{2}$ in test case 1 and $3.5 \mathrm{MW} / \mathrm{m}^{2}$ in test case 2 .

Figure 8 shows the snapshots of static temperature and hydrogen mass fraction in the RDC cross section at height $z=5 \mathrm{~mm}$ above the injector head in test case 4 with $P_{\text {in }}=2.5 \mathrm{MPa}$ (see Table 1 ). It is seen that the mixture ahead of the detonation wave (propagating in a counterclockwise direction) is inhomogeneous in terms of temperature and composition. As a result, the detonation front possesses the $\mathrm{W}$-shape with the leading points at the inner and outer walls and in-between the injection slots. The other important feature evident in Fig. 8 is the existence of premature ignition spots in the tail of the detonation wave. 


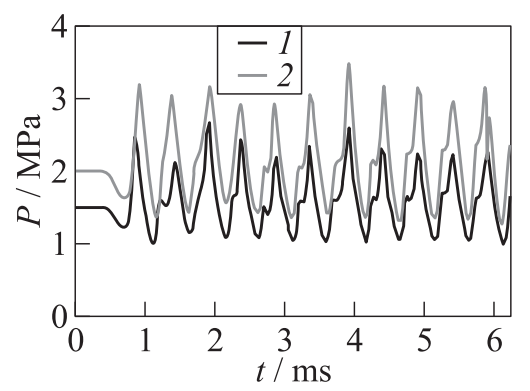

Figure 9 Time histories of the static pressure in the point located in the upstream isolator at $z=-150 \mathrm{~mm}$ for test cases $1(1)$ and $2(2)$

These spots are caused by the mixing of incoming fresh reactants with the hot combustion products.

Of most engineering interest are the flow patterns at the compressor and turbine sides of the isolators. Note that in this study, the authors did not put much effort to properly design the upstream and downstream isolators and considered only simple (tailored) annular geometries to get the estimate for possible flow disturbances.

Figure 9 shows the time histories of the static pressure in a point located in the upstream isolator at $z=-150 \mathrm{~mm}$ for test cases $1(1)$ and $2(2)$. As is seen,
$\%-45 \% P_{\text {in }}$. Such pulsations are accompapressure pulsations attain a value of $40 \%-45 \% P_{\text {in }}$. Such pulsations are accompanied with locally negative axial velocity at the compressor side, which is highly undesirable. Clearly, to avoid compressor stall, special precautions should be undertaken to damp these pressure pulsations. Note that in the course of calculations, the notional particles in the upstream isolator were conditionally treated as nonreacting.

Figure 10 shows the time histories of the static pressure (Fig. 10a) and static temperature (Fig. 10b) in the point located in the downstream isolator at $z=475 \mathrm{~mm}$ for test cases 1 (1) and 2 (2). It is seen that a simple annular downstream isolator tailored to the RDC decreases the amplitudes of static pressure and static temperature pulsations as compared to those in the RDC (see Fig. 4). However, the absolute values of pressure pulsations are still very

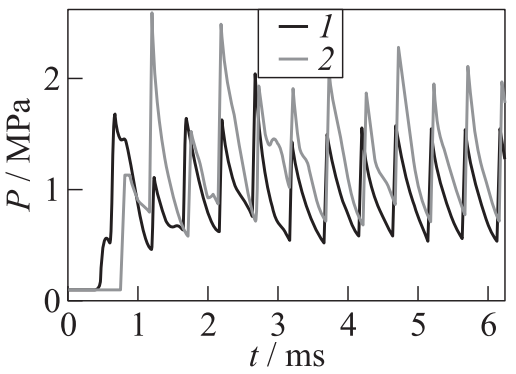

(a)

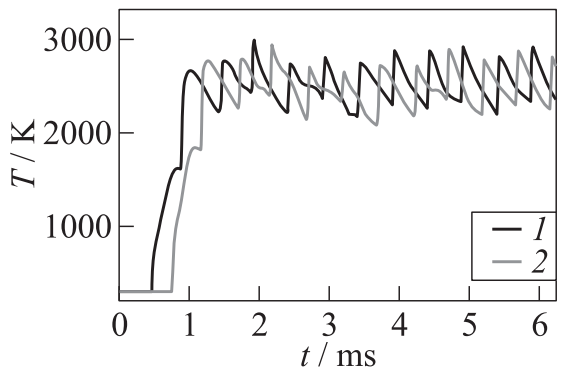

(b)

Figure 10 Time histories of the static pressure $(a)$ and static temperature $(b)$ in the point located at $z=475 \mathrm{~mm}$ in the downstream isolator in test cases 1 (1) and 2 (2) 


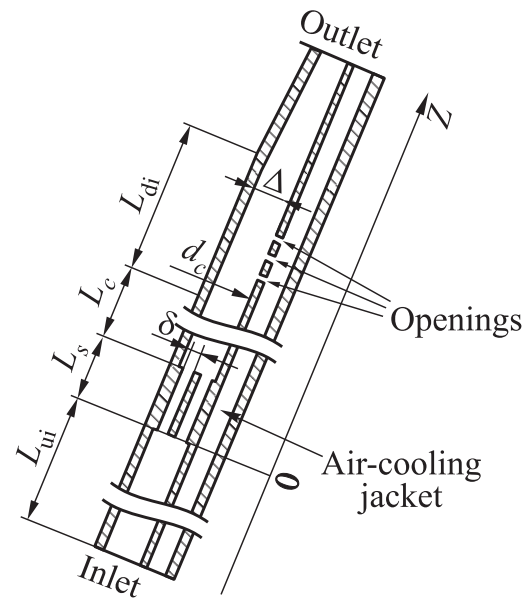

(a)

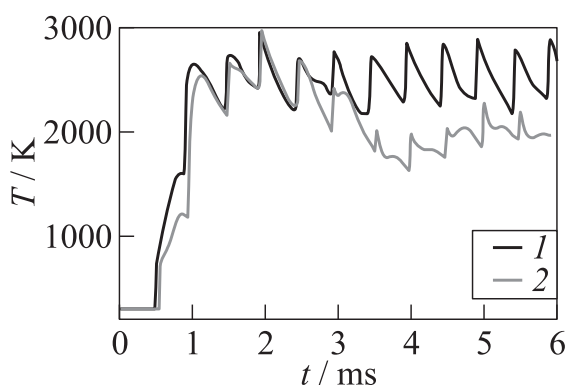

(b)

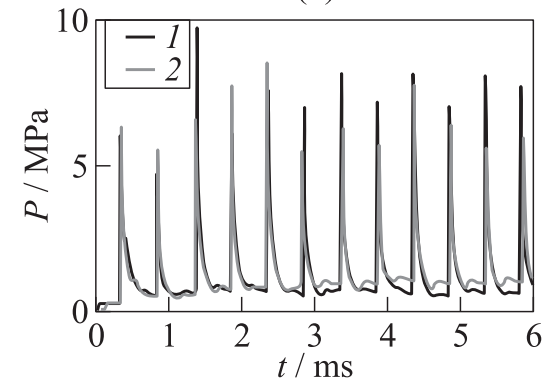

(c)

Figure 11 Schematic of the downstream isolator with air-cooling jacket and openings $(a)$; time histories of the local static temperature $(b)$ and local static pressure in the point located at $z=475 \mathrm{~mm}$ in the downstream isolator $(c)$ without $(1)$ and with (2) air-cooling jacket at $P_{\text {in }}=1.5 \mathrm{MPa}$

high: $\pm 0.5 \mathrm{MPa}$ at $P_{\text {in }}=1.5 \mathrm{MPa}$ and $\pm 0.7 \mathrm{MPa}$ at $P_{\text {in }}=2.0 \mathrm{MPa}$, i. e., on the level of $30 \%-35 \% P_{\text {in }}$. The corresponding local mean temperature pulsations are about $\pm 250 \mathrm{~K}$ at the level of $2500 \mathrm{~K}$, i. e., on the level of $10 \%$. Thus, for damping the pressure and temperature pulsations at the turbine side, the downstream isolator should be properly designed. Moreover, the mean temperature of detonation products remains too high for turbine blades $(\sim 2500 \mathrm{~K})$. Therefore, for decreasing the outlet temperature, the RDC should operate on a fuel-lean mixture and/or the downstream isolator should be equipped with special orifices to mix the detonation products with relatively cold secondary air.

A simple example of such an isolator is shown in Fig. 11a. Here, contrary to the isolator of Fig. $2 b$, a provision is made for the external air-cooling jacket communicating with the downstream isolator through three radial openings each $5 \mathrm{~mm}$ wide. The jacket is assumed to be purged with pure air. As is seen in Fig. $11 b$ plotted for the conditions of test case 1, such a solution allows decreasing both the mean temperature and the amplitude of temperature pulsations at 


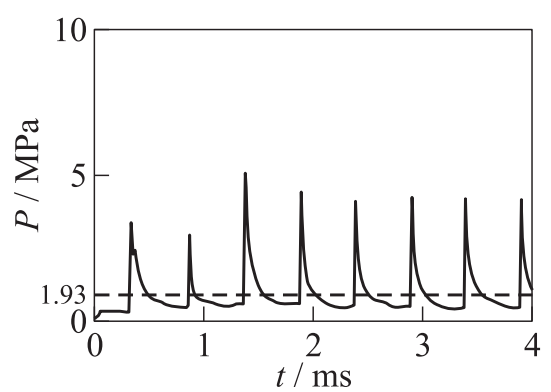

(a)

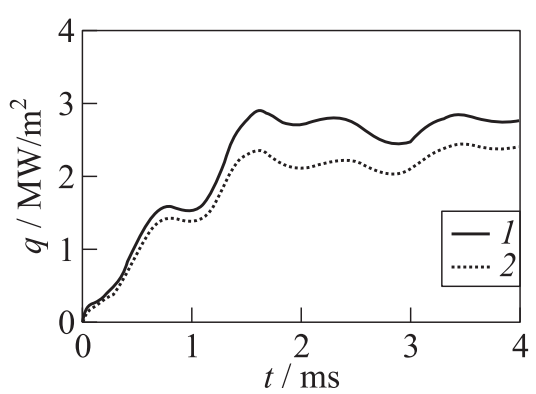

(b)

Figure 12 Time history of the local static pressure at the point located $20 \mathrm{~mm}$ above the injector head in the middle of the RDC gap $(a)$ and total heat flux to the walls of $\mathrm{RDC}$ with the attached isolator $(1-$ inner wall and 2 - outer wall) in test case 5 $\left(P_{\mathrm{in}}=1.0 \mathrm{MPa}\right.$ and $\left.T_{\mathrm{in}}=580 \mathrm{~K}\right)$. Dashed line shows the static pressure averaged over the RDC upon achieving the limit cycle conditions

the isolator outlet. The mean static pressure in the RDC increases (Fig. 11c), which corresponds well to the findings in [5], where the effect of secondary air injection was studied experimentally. Note also that, according to [5], admixing of secondary air to the detonation products results in the increase of the fuel-based specific impulse and in the decrease of the specific fuel consumption. However, these issues are out of the scope of the present paper.

So far, the results of calculations with the injection of cold fuel-air mixture in the RDC (with $T_{\text {in }}=293 \mathrm{~K}$ ) were considered. As a matter of fact, the air in the upstream isolator will possess the elevated temperature due to compression of the ambient air to pressure $P_{\text {in }}$ behind the compressor. The effect of inlet temperature $T_{\text {in }}$ of the stoichiometric hydrogen-air mixture on the RDC operation is demonstrated in Fig. 12. Shown in Fig. 12 are the time histories of the local static pressure (Fig. 12a) and total heat flux to the walls of the RDC with the attached downstream isolator (Fig. $12 b)$ for test case $5\left(T_{\text {in }}=580 \mathrm{~K}\right.$, simple annular geometry, no air-cooling jacket). In this case, the operation process of the RDC was successfully achieved at $P_{\text {in }}=1.0 \mathrm{MPa}$ which was not possible at $T_{\text {in }}=293 \mathrm{~K}$. The static pressure averaged over the RDC (0.93 MPa) and pressure peaks $(\sim 4 \mathrm{MPa})$ in the $\mathrm{RDC}$ decreased as compared to the values relevant to test case 1 . The total heat flux to the walls decreased to about $2.5 \mathrm{MW} / \mathrm{m}^{2}$. As for the level of pressure pulsations in the isolators, it is still on the level of $(40 \%-45 \%) P_{\text {in }}$ for the upstream and $(30 \%-35 \%) P_{\text {in }}$ for the downstream isolator, respectively.

The mass-averaged distributions of static and total pressure, temperature, axial velocity, and density along the $z$-axis of the RDC for test case 5 are presented in Fig. 13. The maximum total pressure in the RDC attained the value 


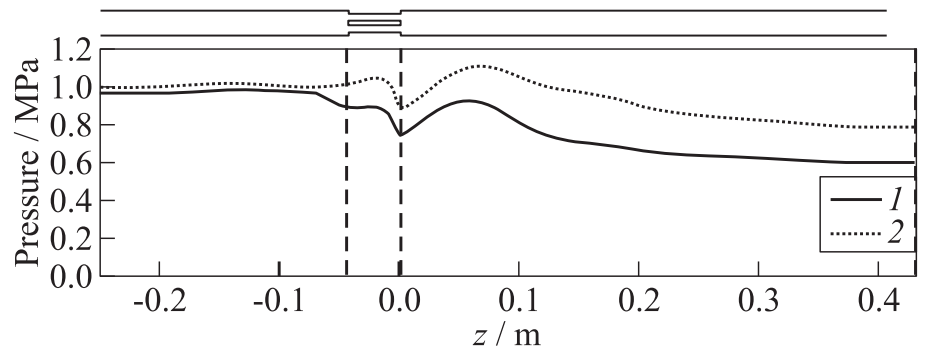

(a)

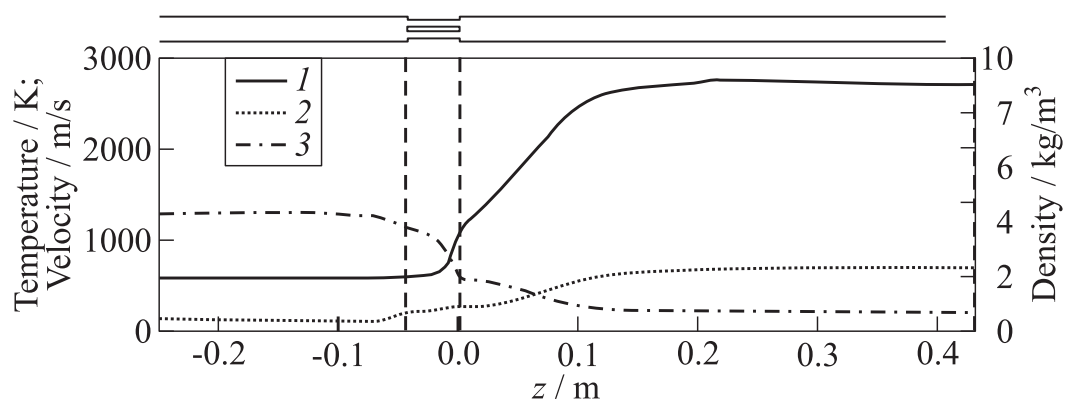

(b)

Figure 13 Mass-averaged distributions of static (1) and total (2) pressure $(a)$ and temperature (1), axial velocity (2), and density (3) (b) in test case 5 at a fixed time after attaining limiting cycle conditions

of $1.11 \mathrm{MPa}$ (Fig. 13a). It is seen from Fig. $13 a$ that after attaining the maximum value, the total pressure drops to about $0.8 \mathrm{MPa}$ due to viscous losses at $\mathrm{RDC}$ walls and irreversible losses in the oblique shock attached to the detonation front. It follows from Fig. $13 b$ that the mass-averaged axial flow velocity in the cylindrical part of the RDC is subsonic. However, further downstream, the flow velocity becomes sonic in the contracting part of the RDC (see Fig. 6a).

Figure $14 a$ shows the geometry of test case $5^{*}$ which differs from test case 5 only by introducing multiple equidistant partitions in the injector face. As is seen from Fig. 14b, this modification of injector face geometry results in the weakening of the shock wave penetrating into the upstream isolator and pressure pulsations in the isolator on the level of $(24 \%-27 \%) P_{\text {in }}$. However, the introduction of partitions leads to additional losses of total pressure and to generation of multiple recirculation zones at the injector face. These zones affect the operation process as they provide a possibility of trapping some combustion products and mixing them with incoming fresh reactants and, thus, introducing an additional mechanism of premature ignition. 


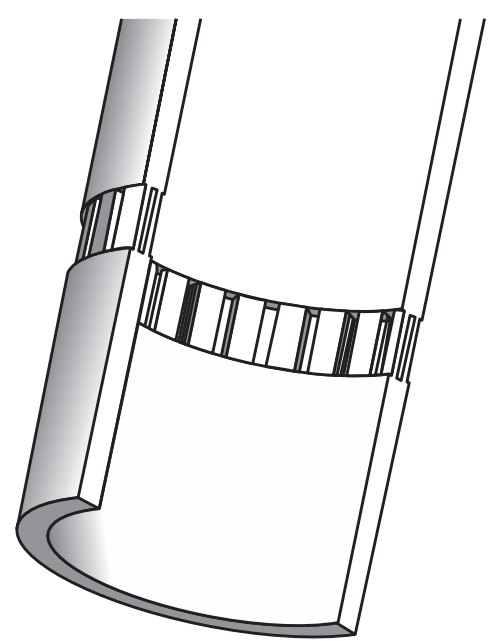

(a)

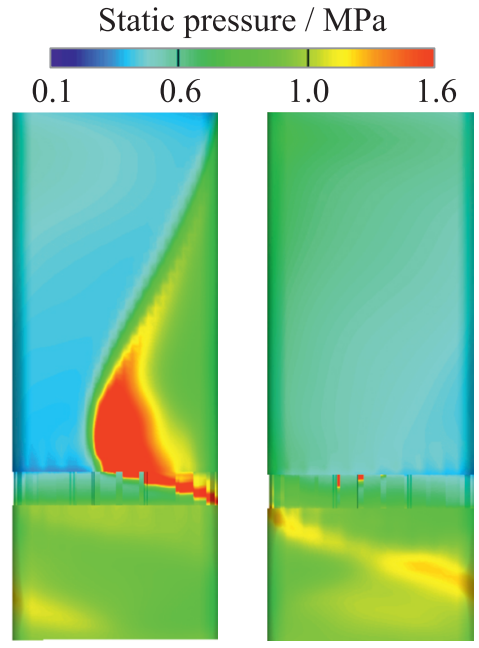

(b)

Figure 14 Schematic of RDC with partitions in the injector face in test case $5^{*}(a)$ and snapshots of mean static pressure $(b)$ in it (two snapshots show the front and back views at the same time instant).

\section{CONCLUDING REMARKS}

The computational tool has been developed for the numerical simulation of the operation process in combustion chambers utilizing rotating detonations. As an example, the annular combustor operating on homogeneous stoichiometric hydrogen-air mixture was considered.

The selected RDC was shown to operate in a stable mode with a single detonation wave rotating at a frequency of $126,000 \mathrm{rpm}(2.1 \mathrm{kHz})$. The domain of detonation existence in the RDC was limited by the condition $1.3 \leq P_{\text {in }}$ $\leq 2.5 \mathrm{MPa}\left(\right.$ at $\left.T_{\text {in }}=293 \mathrm{~K}\right)$. When taking into account the increase of $T_{\text {in }}$ due to adiabatic compression, the lower boundary of the detonation existence domain decreased to $P_{\text {in }} \approx 1.0 \mathrm{MPa}$. In all test cases with the stable operation process, the total pressure in the RDC was higher than $P_{\text {in }}$, thus proving that the RDC is the combustor with pressure gain.

Particular attention was paid to the flow patterns at the compressor and turbine sides of the isolators attached to the RDC, although only simple (tailored) annular geometries of the upstream and downstream isolators were considered. The temperature of the detonation products at the outlet of downstream isolator was about $2500 \mathrm{~K}$ which is too high for turbine blades. For decreasing this temperature, an attempt was made to design the downstream isolator with special 
orifices for mixing the detonation products with relatively cold secondary air. This solution allowed decreasing both the mean temperature and the amplitude of temperature pulsations at the isolator outlet.

The calculations revealed that the level of possible pulsations of the local mean static pressure in the isolators was rather high: $(40 \%-45 \%) P_{\text {in }}$ for the upstream and $(30 \%-35 \%) P_{\text {in }}$ for the downstream isolator (or $(24 \%-27 \%) P_{\text {in }}$ for the injector face with equidistant radial partitions). The possible local mean temperature pulsations in the downstream isolator were shown to attain $10 \%$. Thus, to avoid undesired mechanical loads on compressor and turbine blades and flow instabilities (e.g., compressor stall) special precautions should be undertaken to damp these pulsations.

Both local and total heat fluxes to the walls of the RDC and the downstream isolator were estimated. The local heat fluxes to the RDC walls attained very high values on the level of $16 \mathrm{MW} / \mathrm{m}^{2}$ near the injector head. However, the total heat fluxes, defined as the integral-mean values over inner or outer wall surfaces, were on the level of $2.5-3.5 \mathrm{MW} / \mathrm{m}^{2}$ depending on the injection pressure $P_{\text {in }}$.

The future work will be focused on extending the model to the RDC with separate delivery of fuel and oxidizer and making a provision for more elaborate isolator geometries.

\section{ACKNOWLEDGMENTS}

This work was partly supported by the Russian Foundation for Basic Research (grant 11-08-01297) and the Russian Academy of Sciences (Program \# 26, Combustion and Explosion).

\section{REFERENCES}

1. Voitsekhovskii, B. V. 1959. Stationary detonation. Dokl. USSR Acad. Sci. 129.

2. Bykovskii, F. A., S. A. Zhdan, and E. F. Vedernikov. 2005. Continuous spin detonation in annular combustors. Combust. Explosion Shock Waves 41(4):449.

3. Bykovskii, F. A., S. A. Zhdan, and E. F. Vedernikov. 2006. Continuous spin detonations. J. Propul. Power 22(6):1204.

4. Zhdan, S. A., F. A. Bykovskii, and E. F. Vedernikov. 2007. Mathematical modeling of a rotating detonation wave in a hydrogen-oxygen mixture. Combust. Explosion Shock Waves 43(4):449.

5. Bykovskii, F. A., S. A. Zhdan, and E. F. Vedernikov. 2010. Continuous spin detonation of a hydrogen-air mixture with addition of air into the products and the mixing region. Combust. Explosion Shock Waves 46:1. 
6. Davidenko, D., I. Gokalp, and A. Kudryavtsev. 2008. Numerical study of the continuous detonation wave rocket engine. AIAA Paper No. 2008-2680.

7. Davidenko, D. M., I. Gokalp, and A. N. Kudryavtsev. 2010. Numerical simulation of continuous detonation in a layer of hydrogen-oxygen mixture with periodic conditions. In: Deflagrative and detonative combustion. Eds. G. Roy and S. Frolov. Moscow: TORUS PRESS. 407.

8. Kindracki, J., P. Wolanski, and Z. Gut. 2011. Experimental research on the rotating detonation in gaseous fuels-oxygen mixtures. J. Shock Waves 21(2):75.

9. Schwer, D., and K. Kailasanath. 2011. Numerical investigation of the physics of rotating-detonation-engines. Proc. Combust. Inst. 33(2):2195-202.

10. Hishida, M., T. Fujiwara, and P. Wolanski. 2009. Fundamentals of rotating detonations. J. Shock Waves 19(1):1.

11. Shao, Y.-T., M. Liu, and J.-P. Wang. 2010. Numerical investigation of rotating detonation engine propulsive performance. Combust. Sci. Technol. 182:1586.

12. Pope, S. B. 1985. The PDF method for turbulent combustion. Prog. Energy Combust. Sci. 11:119.

13. Frolov, S. M., V.Ya. Basevich, M. G. Neuhaus, and R. Tatschl. 1997. A joint velocity-scalar PDF method for modeling premixed and nonpremixed combustion. In: Advanced computation and analysis of combustion. Eds. G. D. Roy, S. M. Frolov, and P. Givi. Moscow: ENAS Publs. 537.

14. Frolov, S. M., and V.S. Ivanov. 2010. Combined flame tracking-particle method for numerical simulation of deflagration-to-detonation transition. In: Deflagrative and detonative combustion. Eds. G. Roy and S. Frolov. Moscow: TORUS PRESS. 133.

15. Ivanov, V.S., and S. M. Frolov. 2011. Numerical simulation of the work process and thrust characteristics of an air-breathing pulse detonation engine in supersonic flight conditions. Russ. J. Phys. Chem. B 5(4):597.

16. Frolov, S. M. 2009. Turbulent combustion and localized preflame autoignition of hydrogen-air mixture in an enclosure. In: Teaching materials for the 4th European Summer School on Hydrogen Safety. Corsica, France. Paper \# 12.

17. Basevich, V. Ya, and S. M. Frolov. 2007. Kinetics of 'blue' flames in the gas-phase oxidation and combustion of hydrocarbons and their derivatives. Russ. Chem. Rev. $76(9): 867$. 